\title{
StimuliApp: psychophysical tests on mobile devices
}

\author{
Rafael Marin-Campos ${ }^{a,{ }^{*}}$, Josep Dalmau ${ }^{a, b, c, d, e}$, Albert Compte ${ }^{a}$, Daniel Linares ${ }^{a, b,{ }^{*}}$ \\ a Institut d'Investigacions Biomèdiques August Pi i Sunyer (IDIBAPS), Barcelona, Spain \\ $b$ Centro de Investigación Biomédica en Red de Enfermedades Raras (CIBERER), Barcelona, Spain \\ c Hospital Clínic, University of Barcelona, Barcelona, Spain \\ d Catalan Institution for Research and Advanced Studies (ICREA), Barcelona, Spain \\ e Department of Neurology, University of Pennsylvania, Philadelphia, PA, USA \\ ${ }^{*}$ Corresponding authors
}

\begin{abstract}
Psychophysical tests are commonly carried out running software applications in desktop or laptop computers, but running the software in mobile handheld devices such as smartphones or tablets could have advantages in some situations. Here, we present StimuliApp, an open-source application, in which the user can create psychophysical tests on the iPad and the iPhone by means of a system of menus. A wide number of templates for creating stimuli are available including patches, gradients, gratings, checkerboards, random-dots, texts, tones or auditory noise. Images, videos and audios stored in files could also be presented. The application was developed natively for iPadOS and iOS using the low-level interface Metal for accessing the graphics processing unit, which results in high timing performance.
\end{abstract}

\section{INTRODUCTION}

Psychophysical tests that present stimuli and record responses accurately are essential to study perception and cognition. They are commonly carried out running software applications in desktop or laptop computers, but running the software in mobile handheld devices such as smartphones or tablets could be advantageous in some situations. First, their small size and weight could facilitate their use outside the laboratory in places such as clinical environments (Kalia et al. 2014; Bastawrous et al. 2015; McKendrick et al. 2017; Linares et al., 2020). Second, their touchscreen interface could facilitate their use by people with little experience with more traditional computers. Third, the large number of people owning them, and the ease of installation of applications from common repositories for these devices, could help in the collection of data online. Fourth, they are generally less expensive than desktop or laptop devices.

Psychophysical tests in mobile devices have been carried out with custom applications that implemented specific tests such the assessment of visual acuity (Black et al. 2013), contrast sensitivity (Dorr et al. 2013; Rodríguez-Vallejo et al. 2015; Kollbaum et al. 2014), chromatic contrast sensitivity (Bodduluri et al. 2018) or stereoacuity (Rodríguez-Vallejo et al. 2017), or using an application that allows the sequential presentation of images created off-line (Turpin, Lawson, and McKendrick 2014; McKendrick et al. 2017; Nguyen et al. 2018). To our knowledge, there are no software applications for mobile devices that allow the flexible generation of stimuli 
to create a wide range of psychophysical tests, and this motivated us to create StimuliApp, an open-source application developed natively for iPadOS and iOS, in which the user can create psychophysical tests by means of a system of menus.

\section{DEVELOPMENT}

StimuliApp (www.stimuliapp.com) is a custom application developed natively for Pad and iOS in XCode (Version 11.5). XCode is an integrated development environment (IDE) for developing software using the programming language Swift (Version 5). Swift is a general-purpose programming language built using the high-performance and open source LLVM compiler technology, which transforms Swift code into optimized native code.

Visual stimuli with the exception of texts (which are rendered by the Swift library) are rendered using a function written in Metal. Metal is a low-level hardware-accelerated 3D graphic and computer shader application programming interface (API) based on C++14. Similar to OpenGL, it uses the graphics processing unit (GPU) of the device to perform the calculations in parallel.

Auditory stimuli, taking advantage of the versatility of Swift to implement $C$ code, are coded directly in $\mathrm{C}$ for better performance. They are generated at a high audio rate of $44100 \mathrm{Khz}$.

Touch information is sampled at $120 \mathrm{~Hz}$ in all devices, except for the iPad Pro 11-inch first generation (and later) and the iPad Pro 12.9-inch third generation (and later) in which the sampling rate is $240 \mathrm{~Hz}$.

\section{GRAPHICAL USER INTERFACE (GUI)}

To generate a psychophysical test, StimuliApp uses a graphical user interface (GUI) consisting of a system of menus (Figure 1). Each test is a collection of sections. A section, for example, could be the instructions of the test or each of the trials to be presented. Each section will be a sequence of scenes. For example, the First trial section could have the scenes Fixation, Target and Feedback. Each scene could include several stimuli. For example, the Target scene might consist of the simultaneous presentation of two gratings and one sound.

StimuliApp incorporates several demonstration tests. Tutorials of how these tests have been built can be found at www.stimuliapp.com. To make a copy and modify a demonstration test (or any test created by the user), it is necessary to perform a long tap on the name of the test in the Test menu.

To facilitate the interactive creation of a test, each stimulus, scene or section can be independently previewed. When previewing a scene, it is possible to advance it frame by frame, which could be particularly useful to monitor rapidly changing stimuli. 
Once a test is created, it can be run in the same device by going to the Run test menu. It can also be exported to any other device with StimuliApp installed (for example, a test created on an iPad could be run on an iPhone). To export a test, Export test should be pressed in the Test menu. The user will be able to email a .stimulitest file containing a .json description of the parameters of the test to any device. To import the test in the receiver device, the stimulitest file should be opened with StimuliApp (if StimuliApp does not appear in the list of applications the user should click the More option).

The .stimulitest files, as they contain a .json description, could be edited with any text editor. By default, the files are generated in a single line, but some editors (e.g. atom) have an automatic option to change it to multiple lines. There are also online tools (e.g. jsonformatter) to change a single line to multiple lines. We think, however, that it is easy to get lost in the structure of a .json file and recommend the modification of tests within StimuliApp.

Once a test is run, the results can be accessed in the Results menu. There, the user will find two text files that can be sent by email. One file-with $t x t$ extension-includes information about the settings such as audio rate, screen resolution or frame rate. The other file-with csv extension-consists of a table in which each column is a variable of the test and each row is a section (trial). As this is a typical structure for data analysis, the csv file can be read by standard software to that end such as R, Python, Excel or SPSS.

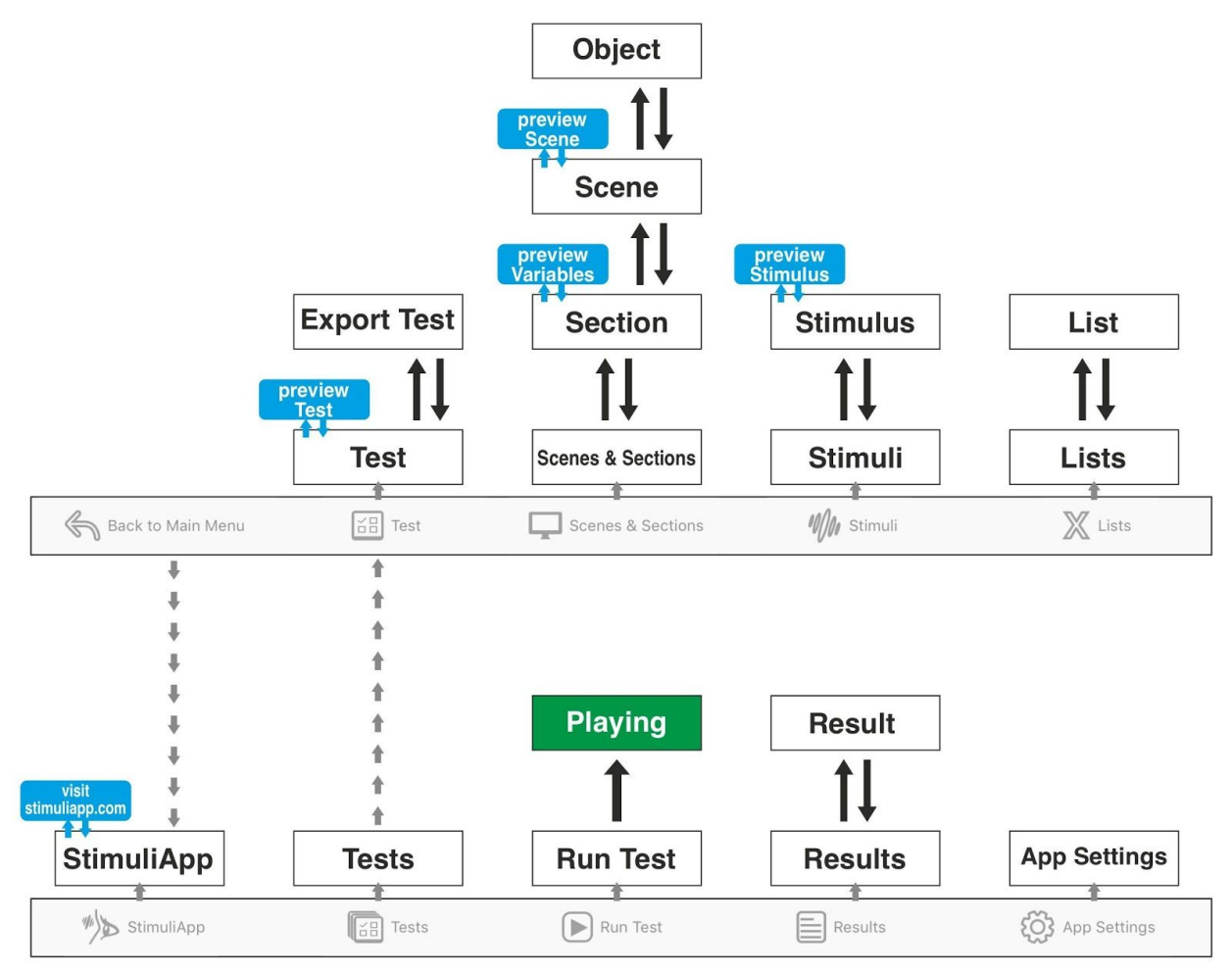

Figure 1. Hierarchy of menus in StimuliApp. 


\section{STIMULI}

StimuliApp offers a large number of templates for creating stimuli commonly used in the study of perception and cognition (Figure 2; Lu and Dosher 2013) including patches, gradients, gratings (Gabors), checkerboards (rectangular or radial), random-dots (linear, radial, expansive), texts, tones or auditory noise. Stimuli can also consist of images, videos and audios stored in files.

Each stimulus has specific properties that define the type of stimulus and general properties such as duration, position, orientation, size, shape, noise filter, color or contrast, which are common. An interesting feature of stimuliApp is that contrast can be manipulated quasi-continuously as the noise-bit method is implemented (Allard and Faubert 2008). All properties can be modified dynamically in the course of the test.

The values of the properties can be specified independently for each stimulus in different units. Sizes and distances, for example, can be specified in pixels, centimeters, inches, or degrees of visual angle, which are calculated taking into account the viewing distance specified by the user and the pixel resolution of the screen of the device, which is directly detected by StimuliApp. Time can be specified in frames or seconds. Luminance is specified in fractions of the maximum brightness of the device. Interestingly, the value of the luminance in $\mathrm{cd} / \mathrm{m}^{2}$ is automatically displayed, since StimuliApp recognizes the model device and incorporates a table with the maximum luminance of each device. The nominal maximum luminance values were retrieved from apple.com and might slightly differ from the displayed values due to variations across series or the time in use of the displays (Caffery, Manthey, and Sim 2016). Importantly, for luminance-and also color-to be displayed consistently, the technologies Auto-Brightness, True Tone and Night Shift of the device should be disabled in System Preferences.

To facilitate the selection of the values across sections (for example, trials) the method of constant stimuli and several adaptive methods are implemented (Kingdom and Prins 2016).

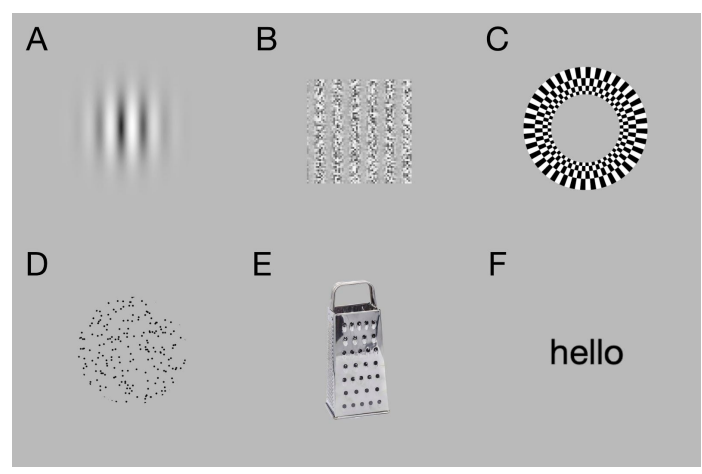

Figure 2. Examples of stimuli that can be presented using StimuliApp. (A) Gabor. (B) Grating with modulated carrier contrast. (C) Radial checkerboard. (D) Random-dots. (E) Image from a file. (F) Text. 


\section{RESPONSES}

Responses can be taps, the movement of a finger or the movement of an electronic pencil on the screen. A virtual keyboard can also be displayed or an external keyboard used.

\section{TIMING}

To compute the RGB values of the stimuli in real-time, the computation should last less than the duration of a frame (16.67 ms for a refresh rate of $60 \mathrm{~Hz}$ and $8.33 \mathrm{~ms}$ for $120 \mathrm{~Hz}$, for example). If the computation lasts longer than a frame, the previous image will be on the screen until the computation is finished (dropped frame).

Using the GPU Frame Capture tool and the Metal API validation tool, we calculated the number of dropped frames for several stimulus tests, which we presented 10 times during $120 \mathrm{~s}$ on several platforms (iPad 6th generation 2018, iPhone X 2017, iPad Pro 1st generation 10.5 inches 2017 at 60 and $120 \mathrm{~Hz}$ ). We tried many simple stimuli typically used in visual psychophysics and all resulted in zero dropped frames in all platforms. We were able to get dropped frames only when we displayed complex stimuli on the iPad Pro at $120 \mathrm{~Hz}$ (Figure 3). All of these stimuli resulted in zero dropped frames in the other platforms or when the iPad Pro was run at $60 \mathrm{~Hz}$ (Figure 3).

When a test is previewed or run, and one or more frames are dropped, a report is generated indicating the duration of the dropped frames. This information could be used to reduce the computational costs of the test.

To assess the accuracy and precision reproducing sounds, we presented 1000 sounds in StimuliApp specifying a duration of $100 \mathrm{~ms}$ each. Connecting an output jack to an oscilloscope (Tektronix TDS 1012), we measured the generated durations and found a mean duration of 100 $\mathrm{ms}$ and a standard deviation of less than $1 \mathrm{~ms}$.

To assess audiovisual synchrony, we presented 1000 times a visual and an auditory signal of $100 \mathrm{~ms}$ of duration specifying the same time onset in StimuliApp. Connecting an output jack and a photodiode to the oscilloscope, we measured the generated onset asynchronies and found that the mean asynchrony depended on the device and was between $-10 \mathrm{~ms}$ and $10 \mathrm{~ms}$ (this value could be specified in StimuliApp to correct it). The standard deviation was less than $1 \mathrm{~ms}$. 


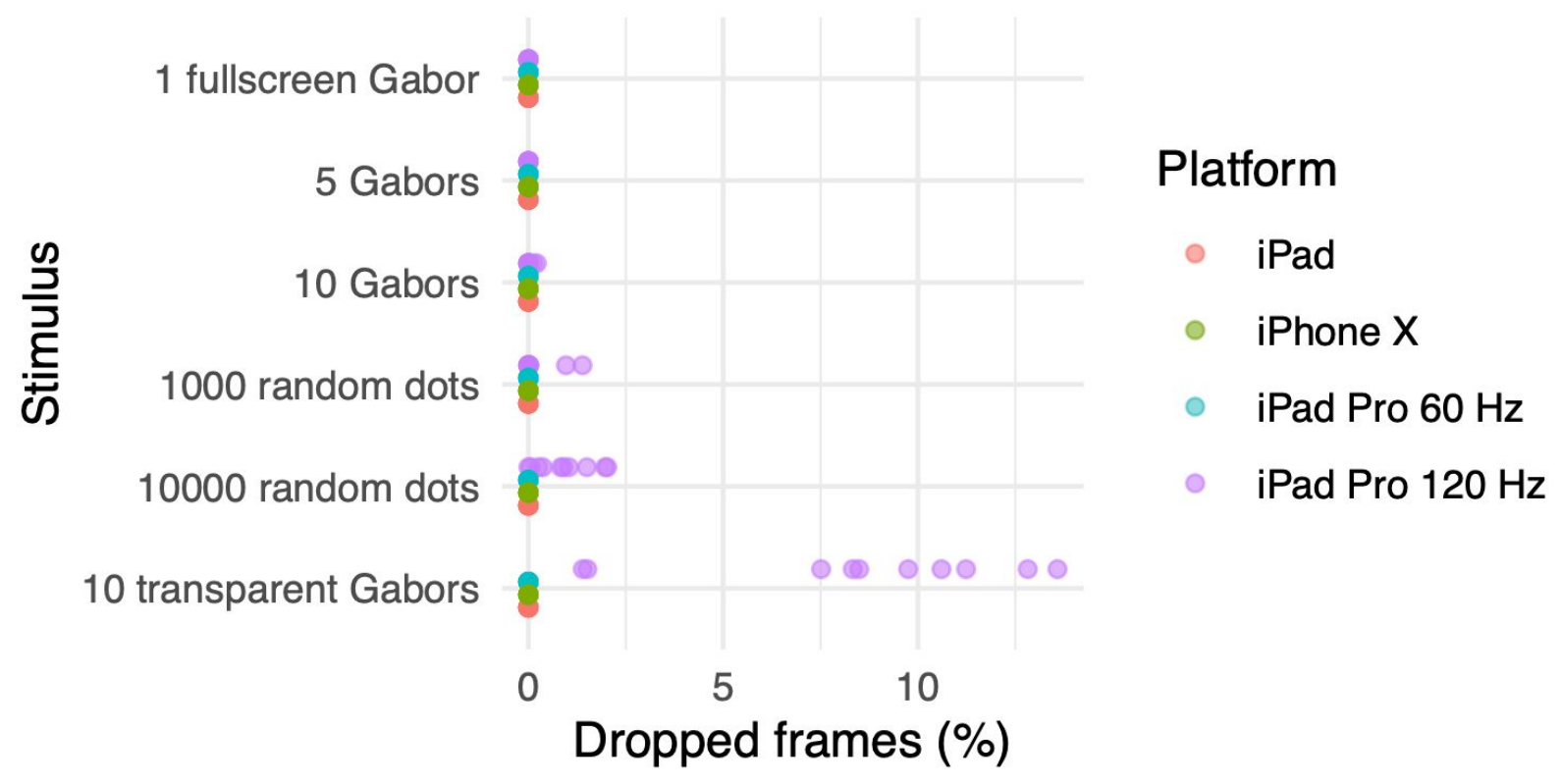

Figure 3. Percentage of dropped frames for different stimuli in different platforms. Each dot indicates the percentage of dropped frames when we displayed the stimulus for $120 \mathrm{~s}$. We presented each stimulus 10 times. The Gabors were $300 \times 300$ pixels (excepting the fullscreen Gabor). The transparent Gabors had quasi-continuous contrast using the noise-bit method (Allard and Faubert 2008).

\section{EXTENDING STIMULIAPP}

Developers can add stimuli to StimuliApp by downloading its open source code and compiling it including a new independent Metal function for each new stimulus, and one descriptive class in Swift with the description of its new parameters. These new parameters will be added to the general parameters common to all stimuli such as size, contrast, angle, noise and temporal changes. For more information visit https://github.com/marinraf/StimuliApp.

\section{WORKFLOW TO COLLECT DATA USING STIMULIAPP: EXAMPLES}

The first example describes a situation where the test is created and run on the same device. This possibility could be useful to administer tests in person. An experimenter installs StimuliApp on an iPad and uses the application to create a new test. Once the test is created, the experimenter goes to the Run section of the application and hands the iPad to a participant. After the participant finishes the test, the experimenter goes to the Results section and sends the two output data files with the results to her email.

The second example describes a situation where the test is created on one device and run on another device. This possibility could be useful to administer tests remotely. An experimenter installs StimuliApp on an iPhone and uses the application to create a new test. Once the test is created, the experimenter exports the test (see GUI section) and sends it by email to the 
participant. The participant installs StimuliApp on her iPad and then opens the file sent by the experimenter with StimuliApp. Then, the participant goes to the Run section and runs the test. After finishing, the participant goes to the Results section and sends the two files with the results to the experimenter.

\section{TUTORIAL}

In this section, we describe how to build a simple test to measure orientation discrimination using the method of the constant stimuli (Kingdom and Prins 2016). Each trial will consist of a fixation cross followed by a grating with a small clockwise or counter-clockwise rotation relative to the vertical orientation. The participant will need to tap the right or left side of the screen to indicate clockwise or counterclockwise rotation, respectively. Further tests with more detailed information including screen captures can be found at www.stimuliapp.com.

First, we create a fixation cross:

- Go to the Test menu (Figure 1) and press new test.

- Go to the Stimuli menu (Figure 1) and press new stimulus.

- Change the stimulus shape to cross.

Then, we include the fixation as the first scene of a section:

- Go to the Scenes \& Sections menu (Figure 1) and press new section.

- Within that section, press new scene.

- Within the scene, press new object and select stimulus1.

- Press durationValue and change the scene duration to 0.5 seconds.

- Go to the Test menu, press firstSection and select section1.

Then, we create the grating:

- Go to the Stimuli menu and press Stimuli in the top-left corner to go to the home screen of the Stimuli menu. Press new stimulus.

- Press type and change the stimulus type to grating.

- Change the stimulus shape to ellipse.

Then, we include the grating as the second scene within the section:

- Go to the Scenes \& Sections menu and press section1 in the top-left corner to go to the Sections menu. Press new scene.

- Within the scene, press new object and select stimulus2.

Then, we tell StimuliApp to present the stimulus multiple times with a different orientation each time:

- Go to the Stimuli menu, press the property gratingRotation and change it to variable.

- Go to the Lists menu, press new list and select new list of numeric values.

- Press add linear sequence. For the First value, Last value and Number of values assign the numbers $-0.03,0.03$ and 7 , respectively (the first two are angles in radians). 
- Go to the Scenes \& Sections menu and press section1 (in the top-left corner of the screen).

- Press the newly created property scene2_object1_gratingRotation and assign the list created above.

- From the selectionMethod select all values in random order.

- Press section 1 and change the number of repetitions to 20 .

Then, we allow the user to make responses:

- Go to scene2, press response and add a left or right response.

Finally, we run the test:

- Go to the Main menu and then to the Run test menu. Press Run: test1.

The text file with the results can be found in the Results menu.

\section{DISCUSSION}

StimuliApp is an open-source application that allows the generation of a wide range of psychophysical tests on mobile devices. As it has been developed natively for iPadOS and iOS, the supported mobile devices are the iPad and the iPhone. We decided to program the application natively to try to achieve high timing performance, and our measurements suggest that this was achieved for stimulus presentation as StimuliApp, for most situations, results in zero dropped frames. Future research should also test the temporal precision of touch responses. We chose the Apple ecosystem because it has a relatively small number of available models, which facilitates the testing and that the application, by recognizing the model, can present about the same stimuli independently of the device. The value of the luminance, for example, is directly displayed in $\mathrm{cd} / \mathrm{m}^{2}$ without the need of a photometer.

For desktop and laptop computers, the flexible generation of psychophysical tests could be performed among others using the platform-independent packages Psychtoolbox (Kleiner, Brainard, and Pelli 2007) and Psychopy (Peirce 2007), both resulting in high timing performance (Bridges et al. 2020). Psychopy offers the possibility of generating psychophysical tests using a GUI without the necessity of coding, similar to StimuliApp. It also allows the execution of psychophysical tests on the web-browser, although a decrease in timing performance has been reported (Bridges et al. 2020; Anwyl-Irvine et al. 2020). Running Psychopy on the browser of a mobile device could be an alternative to creating psychophysical tests on mobile devices.

In the Introduction section we described a number of advantages of using mobile devices to run psychophysical tests, but there are also limitations. First, psychophysical tests have been traditionally conducted using CRT monitors, which have better spatiotemporal properties that the LCD screens incorporated in mobile devices (Ghodrati, Morris, and Price 2015; Elze and Tanner 2012; Packer et al. 2001). In a previous study, however, we compared motion sensitivity for very brief stimuli presenting them on an iPad (using an earlier version of StimuliApp) or in a 
CRT monitor (using Psychopy) and found comparable values (Linares et al. 2018). Second, chin rests-which are often used when the experimental set-up consists of an external monitor connected to a desktop computer-could be hardly combined with mobile devices, hindering the maintenance of a constant viewing distance. To ameliorate this issue, in our previous studies (Linares et al. 2018, 2020) the experimenter monitored that participants hold the same approximate position during the test. A future solution to this issue, could be the use of a facial recognition system like Face ID to measure the viewing distance and alert the participant if it changes during the test. This technology might be also used to track eye movements. Third, many mobile devices incorporate glossy screens, whose glare might be superimposed on the visual stimuli. To minimize this problem, the participant should run the test in a location where reflections are minimized.

Tablets and smartphones are increasingly used to acquire data in behavioural sciences (Miller 2012; Woods et al. 2015). Here, we present an application for iPadOS and iOS devices to create psychophysical tests with high temporal precision.

\section{OPEN PRACTICES STATEMENT}

The application described here is open source. None of the data or materials for the experiments reported here is available, and none of the experiments was preregistered.

\section{ACKNOWLEDGMENTS}

This work was supported by the Fundación Alicia Koplowitz, Fundació CELLEX, La Caixa Foundation Health Research, CIBERER (Refs: 15/00010), the Spanish Ministry of Science, Innovation and Universities and European Regional Development Fund (Refs: BFU2015-65315-R, RTI2018-094190-B-I00), the Generalitat de Catalunya (PERIS-ICT SLT002/16/00338; PERIS SLT006/17/00362) and Instituto Carlos III/FEDER (Refs: FIS 17/00234, PIE 16/00014), and by the CERCA Programme/Generalitat de Catalunya. This work was developed at the building Centro Esther Koplowitz, Barcelona.

\section{REFERENCES}

Allard, Rémy, and Jocelyn Faubert. 2008. "The Noisy-Bit Method for Digital Displays: Converting a 256 Luminance Resolution into a Continuous Resolution." Behavior Research Methods 40 (3): 735-43.

Anwyl-Irvine, Alexander L., Edwin S. Dalmaijer, Nick Hodges, and Jo Evershed. 2020. "Online Timing Accuracy and Precision: A Comparison of Platforms, Browsers, and Participant's Devices." https://doi.org/10.31234/osf.io/jfeca.

Bastawrous, Andrew, Hillary K. Rono, lain A. T. Livingstone, Helen A. Weiss, Stewart Jordan, Hannah Kuper, and Matthew J. Burton. 2015. "Development and Validation of a Smartphone-Based Visual Acuity Test (Peek Acuity) for Clinical Practice and Community-Based Fieldwork." JAMA Ophthalmology 133 (8): 930-37.

Black, J. M., R. J. Jacobs, G. Phillips, L. Chen, E. Tan, A. Tran, and B. Thompson. 2013. “An 
Assessment of the iPad as a Testing Platform for Distance Visual Acuity in Adults." BMJ Open 3 (6). https://doi.org/10.1136/bmjopen-2013-002730.

Bodduluri, Lakshmi, Mei Ying Boon, Malcolm Ryan, and Stephen J. Dain. 2018. "Normative Values for a Tablet Computer-Based Application to Assess Chromatic Contrast Sensitivity." Behavior Research Methods 50 (2): 673-83.

Bridges, David, Alain Pitiot, Michael R. MacAskill, and Jonathan Peirce. 2020. "The Timing Mega-Study: Comparing a Range of Experiment Generators, Both Lab-Based and Online." https://psyarxiv.com/d6nu5/download?format=pdf.

Caffery, Liam J., Kenneth L. Manthey, and Lawrence H. Sim. 2016. "The Effect of Time in Use on the Display Performance of the iPad." The British Journal of Radiology 89 (1063): 20150657.

Dorr, Michael, Luis A. Lesmes, Zhong-Lin Lu, and Peter J. Bex. 2013. "Rapid and Reliable Assessment of the Contrast Sensitivity Function on an iPad." Investigative Ophthalmology \& Visual Science 54 (12): 7266-73.

Elze, Tobias, and Thomas G. Tanner. 2012. "Temporal Properties of Liquid Crystal Displays: Implications for Vision Science Experiments." PloS One 7 (9): e44048.

Ghodrati, Masoud, Adam P. Morris, and Nicholas Seow Chiang Price. 2015. "The (un)suitability of Modern Liquid Crystal Displays (LCDs) for Vision Research." Frontiers in Psychology 6: 303.

Kalia, Amy, Luis Andres Lesmes, Michael Dorr, Tapan Gandhi, Garga Chatterjee, Suma Ganesh, Peter J. Bex, and Pawan Sinha. 2014. "Development of Pattern Vision Following Early and Extended Blindness." Proceedings of the National Academy of Sciences of the United States of America 111 (5): 2035-39.

Kingdom, F. A. A., and N. Prins. 2016. Psychophysics: A Practical Introduction. Elsevier Science.

Kleiner, Mario, David Brainard, and Denis Pelli. 2007. "What's New in Psychtoolbox-3?" https://pure.mpg.de/rest/items/item_1790332/component/file_3136265/content.

Kollbaum, Pete S., Meredith E. Jansen, Elli J. Kollbaum, and Mark A. Bullimore. 2014. "Validation of an iPad Test of Letter Contrast Sensitivity." Optometry and Vision Science: Official Publication of the American Academy of Optometry 91 (3): 291-96.

Linares, Daniel, Silvia Amoretti, Rafael Marin-Campos, Andre Sousa, Laia Prades, Josep Dalmau, Miquel Bernardo, and Albert Compte. 2020 "Perceptual Spatial Suppression and Sensitivity for Motion Are Weakened in Schizophrenia." Schizophrenia Bulletin Open, in press.

Linares, Daniel, Rafael Marin-Campos, Josep Dalmau, and Albert Compte. 2018. "Validation of Motion Perception of Briefly Displayed Images Using a Tablet." Scientific Reports 8 (1): 16056.

Lu, Zhong-Lin, and Barbara Dosher. 2013. Visual Psychophysics: From Laboratory to Theory. MIT Press.

McKendrick, Allison M., Yu Man Chan, Algis J. Vingrys, Andrew Turpin, and David R. Badcock. 2017. "Daily Vision Testing Can Expose the Prodromal Phase of Migraine." Cephalalgia: An International Journal of Headache, January, 333102417741130.

Miller, Geoffrey. 2012. "The Smartphone Psychology Manifesto." Perspectives on Psychological Science: A Journal of the Association for Psychological Science 7 (3): 221-37.

Nguyen, Bao N., Sui-Ann Hew, John Ly, Hee-Young Shin, Jessica C. Wong, Emily Yeung, and Allison M. McKendrick. 2018. "Acute Caffeine Ingestion Affects Surround Suppression of Perceived Contrast." Journal of Psychopharmacology 32 (1): 81-88.

Packer, O., L. C. Diller, J. Verweij, B. B. Lee, J. Pokorny, D. R. Williams, D. M. Dacey, and D. H. 
Brainard. 2001. "Characterization and Use of a Digital Light Projector for Vision Research." Vision Research 41 (4): 427-39.

Peirce, Jonathan W. 2007. "PsychoPy—Psychophysics Software in Python." Journal of Neuroscience Methods 162 (1-2): 8-13.

Rodríguez-Vallejo, Manuel, Vicente Ferrando, Diego Montagud, Juan A. Monsoriu, and Walter D. Furlan. 2017. "Stereopsis Assessment at Multiple Distances with an iPad Application." Displays 50 (December): 35-40.

Rodríguez-Vallejo, Manuel, Laura Remón, Juan A. Monsoriu, and Walter D. Furlan. 2015. "Designing a New Test for Contrast Sensitivity Function Measurement with iPad." Journal of Optometry 8 (2): 101-8.

Turpin, Andrew, David J. Lawson, and Allison M. McKendrick. 2014. "PsyPad: A Platform for Visual Psychophysics on the iPad." Journal of Vision 14 (3): 16.

Woods, Andy T., Carlos Velasco, Carmel A. Levitan, Xiaoang Wan, and Charles Spence. 2015. "Conducting Perception Research over the Internet: A Tutorial Review." PeerJ 3 (July): e1058. 